(4) acute atorvastatin-treated group with Ipost. T2DM were induced with streptozotocin $(40 \mathrm{mg} / \mathrm{kg}$, i.p.) after 4 -week high-fat diet. All rat hearts were allowed to stabilise for $30 \mathrm{~min}$ followed by 30 min of ischaemia and 120 min of reperfusion by the Langendorff technique, and they were divided into regional ischaemia (induced by ligation the left main artery) and global ischaemia (induced by clamping perfusion circuit). Postconditioning was achieved by six cycles of $10 \mathrm{~s}$ ischaemia-reperfusion periods after ischaemia. During reperfusion, the functional parameter, the peak rate of pressure development $\left(+\mathrm{dP} / \mathrm{dt}_{\max }\right)$, was recorded at 5, 30, 60, 90 and $120 \mathrm{~min}$ in global-ischaemia protocols, and its recovery was expressed as a percentage of initial preischaemic values. At the end of perfusion, infarct area and risk zone of stained hearts were measured, and standard Western blot analysis was performed.

Results Postconditioning markly reduced infart size (the ratio of infarct area and risk zone, \%) and improved the values of $+\mathrm{dP} / \mathrm{dt}_{\max }$ (data not shown) in standard-diet group $(22.35 \pm 4.50 \%$ vs $44.51 \pm 3.53 \%, \mathrm{p}<0.05)$, but failed in T2DM group $(58.06 \pm 5.37 \%$ vs $57.38 \pm 5.11 \%, p>0.05)$. Acute atorvastatin treatment couldn't decrease ischaemia-reperfusion injury in the healthy and DM rat hearts $(41.65 \pm 4.41 \%$ vs $44.51 \pm 3.53 \%$, and $55.85 \pm 4.79 \%$ vs $57.38 \pm 5.11 \%, \mathrm{p}>0.05)$. However, this short-term statin therapy didn't affect infarct size-limiting and contractile dysfunction-recovering of Ipost in healthy rat hearts $(24.11 \pm 4.08 \%$ vs $22.35 \pm 4.50 \%$, $\mathrm{p}>0.05)$, it restored the protection of Ipost in DM ones $(35.65 \pm 4.93 \%$ vs $58.06 \pm 5.37 \%, \mathrm{p}<0.05)$. Western blot analysis revealed that the phosphorylation of Akt Ser ${ }^{473}$ and eNOS Ser ${ }^{1177}$ increasing was indicated in Ipost group, but not in Ipost T2DM group. Acute atorvastatin treatment slightly increased the phosphorylated expression of Akt and eNOS both in healthy and in T2DM rats. 3-day statin application didn't further increase the phosphorylated Akt and eNOS levels of Ipost in healthy rats, but achieve the largest increasing in T2DM rats. Conclusions Acute application of atorvastatin show cardioprotective effect in neither healthy rats nor in T2DM ones, and did not interfere with the protection of postconditioning in normal-diet rats, but could restore the infarct size-limiting and contractile dysfunction-reducing of Ipost in the diabetic rats. This study demonstrated that the mechanism was involved in increasing phosphorylation of Akt and eNOS.

\section{E0079 PRESERVATION OF THE CARDIAC FUNCTION IN INFARCTED RAT HEARTS BY THE TRANSPLANTATION OF ADIPOSE- DERIVED STEM CELLS WITH INJECTABLE FIBRIN SCAFFOLDS}

doi:10.1136/hrt.2010.208967.79

Zhang Xuelian, Ma Yitong, Ma Xiang, Adila Azhati, Wang Baozhu, Chen Liu Fen. Department of Cardiology of The First Affiliated Hospital Xinjiang Medical University

Objective Cell-based therapy can improve cardiac function but is limited by the low cell retention and survival within ischaemic tissues. Injectable cardiac tissue engineering aims to support cellbased therapies and enhance their efficacy for cardiac diseases. Our research is devoted to studying the usefulness of the combination of fibrin glue (as scaffold) and adipose-derived stem cells (ADSCs) to treat myocardial infarction.

Methods The rat ADSCs were isolated from subcutaneous adipose tissues. The surface phenotype of these cells was analysed by flow cytometry. Fibrin glue was then co-injected with ADSCs into the left ventricular wall of rat infarction models. The structure and functional consequences of transplantation were determined by detailed histological analysis and echocardiography.

Results Most cultured ADSCs expressed CD105 and CD90, and negative for CD34 and CD45. After injection, both the 24h-cell retention and 4-week graft size were significantly higher and larger in the Fibrin + ADSCs group than those of the ADSCs group alone $(p<0.01)$. The ADSCs could differentiate into cardiomyocyte-like, endothelial and vascular smooth muscle cells in vivo. The heart function improved significantly in the Fibrin+ADSCs group compared to that of the ADSCs group 4 weeks after transplantation $(p<0.01)$. In addition, the arteriole densities within the infarcted area improved significantly in the Fibrin+ADSCs group compared to those in the ADSCs group, 4 weeks after transplantation $(p<0.01)$.

Conclusions The ADSCs with fibrin glue has the therapeutic potential to improve the function of infarcted hearts. The method of in situ injectable tissue engineering combining fibrin glue with ADSCs is promising clinically.

\section{e0080 THE CYP2J2 G50T POLYMORPHISM AND THE RISK OF CORONARY ARTERY DISEASE IN HAN CHINESE POPULATION}

doi:10.1136/hrt.2010.208967.80

Fu Zhenyan, Xie Xiang, Ma Yitong, Yang Yining, Yuan Shan, Wang Lei. Department of Cardiovascular Medicine The First Affiliated Hospital Xinjiang Medical University

Objective CYP2J2 is the major enzyme responsible for the formation of epoxyeicosatrienoic acids (EETs) in the heart, the EETs are potent endogenous vasodilators and inhibitors of vascular inflammation. The CYP2J2 G-50T polymorphism has been shown to be associated with increased risk of coronary artery disease (CAD) via lower plasma concentrations of EETs, while there are currently some population-based studies which found controversial results. The aim of the study was to assess associations between the G-50T polymorphism of CYP2J2 and CAD via a case-control study in the Han population of China.

Methods There were $249 \mathrm{CAD}$ patients and 243 age-matched control subjects genotyped for the CYP2J2 G-50T polymorphism. The data were assessed for three separate groups: the total subjects, men and women.

Results In total, the distribution of the dominant model of G-50T promoter $(G / G$ versus $G T+T T)$ was significantly lower in $C A D$ patients than control subjects $(p=0.048)$, and simultaneously the $T$ allele was significantly lower in $\mathrm{CAD}$ patients than control subjects $(p=0.037)$. However, logistic regression analysis failed to show that the GT+TT genotype was a protective factor for CAD (OR 0.477, $95 \%$ CI 0.175 to $1.299 ; p=0.148)$. We investigated the synergistic effect between $\mathrm{G} / \mathrm{T}+\mathrm{T} / \mathrm{T}$ genotype and no smoking or no DM, finally results showed $\mathrm{G} / \mathrm{T}+\mathrm{T} / \mathrm{T}$ genotype and no smoking had a tendency to be a synergistic effect ( $\mathrm{G} / \mathrm{T}+\mathrm{T} / \mathrm{T}$ genotype+no smoking $\mathrm{OR}=0.296$ vs $\mathrm{G} / \mathrm{T}+\mathrm{T} / \mathrm{T}$ genotype + smoking $\mathrm{OR}=0.730)$, while lacked sufficient statistical power $(p=0.127, p=0.663$, respectively). Conclusions In presence of other risk factors, the CYP2J2 G-50T failed to show a significant association with coronary artery disease in Han population of China. However, since our result is close to the border of significance, further research based on the larger, prospective researches are necessary.

\section{e0081 ASSOCIATION OF GENETIC POLYMORPHISMS OF SAA1 AND SAA2 WITH CORONARY ARTERY DISEASE IN CHINESE HAN POPULATION}

doi:10.1136/hrt.2010.208967.81

Xie Xiang, Ma Yitong, Yang Yining, Fu Zhenyan, Li Xiaomei, Huang Ding, Ma Xiang, Chen Bangdang, Liu Fen. Department of Cardiovascular Medicine The First Affiliated Hospital Xinjiang Medical University

Background Both Low plasma HDL cholesterol (HDL-C) and inflammatory responses are associated with an increased risk of coronary artery disease (CAD). Serum amyloid A protein (SAA) is 
not only an inflammatory factor but also an apolipoprotein that can replace apolipoprotein $\mathrm{A} 1$ (apoA1) as the major apolipoprotein of HDL. However, the relationship between genetic polymorphisms of $\mathrm{SAA}$ and $\mathrm{CAD}$ remains unclear.

Methods 4 Single Nucleotide Polymorphisms (SNPs) (rs12218, rs1059559, rs2229338, and rs2468844) of SAA1 and SAA2 gene were genotyped in $1580 \mathrm{CAD}$ patients and 1914 age- and sex-matched controls by the use of PCR-restriction fragment length polymorphism (PCR-RFLP) analysis.

Results The CC genotype and C allele of rs12218 and the GG genotype and $\mathrm{G}$ allele of rs2468844 were more common in the CAD patients than in the control subjects, respectively (all $\mathrm{p}<0.001$ ). After adjusted for diabetes mellitus, hypertension, smoking, drinking and lipid disorders by use of logistic regression, the SNPs rs12218 (OR=5.906, 95\% CI 2.877 to $12.124, p<0.001)$ and rs2468844 (OR=4.102, 95\% CI 2.018 to 8.129 , p < 0.001) still differed significantly between the CAD patients and control subjects.

Conclusion These data suggest that genetic polymorphisms of SAA1/2 gene significantly increased the risk of CAD in a Chinese Han population.

\section{e0082 MOLECULAR IMAGING OF APELIN ON SURVIVAL AND FUNCTION OF MESENCHYMAL STEM CELLS IN HINDLIMB MICE}

doi:10.1136/hrt.2010.208967.82

Dong Liang, Weijie Li, Shuang Li, Rongqing Zhang, Lina Gao, Shunming Zhu, Yabin Wang, Haichang Wang, Feng Cao. Department of Cardiology, Xijing Hospital, Fourth Military Medical University, Xi'an, Shaanxi, China

Objective This study was designed to evaluate the contribution of apelin to the therapeutic efficacy of mesenchymal stem cells in hindlimb ischaemia mice.

Methods Mesenchymal stem cells (MSC) expressing firefly luciferase (Fluc) were isolated from B-actin-luc mice and characterised by flow cytometry and bioluminescence imaging (BLI). Male FVB mice underwent femoral artery ligation and received MSC $\left(1 \times 10^{6}\right)$ or MSC with Apelin intra-quadriceps femoris muscle injection. Cell survival was imaged by BLI. Angiogenesis was assessed by immunohistochemisty method. The expressions of AKT and pAKT after cellular therapy were analysed by Western blot.

Results Fluc expression correlated with cell number in all groups. In vivo BLI revealed acute donor cell death of MSC within 2 weeks after transplantation. By contrast, signals of injected cells were still present after 4 weeks in the MSC with apelin group. Immunohistochemisty showed more angiogenesis in the MSC with Apelin group compared to MSC $(p<0.05)$. In vitro apelin treatment of MSC exposed to hypoxia increased cell proliferation. Moreover, considerable increases in phosphorylation of Akt were found in MSC pretreated with apelin.

Conclusions Apelin has beneficial effects on the therapeutic efficacy and survival maintenance of mesenchymal stem cells in hindlimb ischaemia and might constitute an important therapy target in cardiovascular disease.

\section{e0083 MEMBRANE TYPE 1 MATRIX METALLOPROTEINASE ACTIVATION IS ENHANCED BY LOW SHEAR STRESS THROUGH INTEGRIN PATHWAY IN APOE-/- MICE}

\section{doi:10.1136/hrt.2010.208967.83}

Chen Liang, Cai Xiaojun, Li Xuan, Liu Xiaoling, Zhang Yun, Zhang Mei. Shandong University Qilu Hospital

Objective Low shear stress and matrix metalloproteinase are involved in atherogenesis and plaque stability. The aim of this study wad to explore the relation and possible mechanism of membrane type-1 matrix metalloproteinase (MT1-MMP) and low shear stress (LSS).

Methods 80 male apoE-/- mice were fed a high-fat diet, and lesions in the left carotid artery were induced by perivascular placement of constrictive collars.

Results Ultrasound-determined shear stress was significantly lower in the left carotid artery than in the right artery $(5.61 \pm 0.72$ vs $\left.9.76 \pm 1.48 \mathrm{~N} / \mathrm{m}^{2}, \mathrm{p}<0.01\right)$. Confocal microscopy and dual staining revealed higher MT1-MMP expression in left common carotid artery. Cultured human umbilical vein endothelial cells (HUVECs) exposed to $0.4 \mathrm{~N} / \mathrm{cm}^{2}$ shear stress (LSS) and $1.2 \mathrm{~N} / \mathrm{cm}^{2}$ shear stress (PSS). HUVECs subjected to LSS showed a time-dependent increase in MT1-MMP mRNA level. MT1-MMP mRNA level was downregulated under PSS at 3 and $5 \mathrm{~h}$. MT1-MMP protein showed no change in expression at 1-h LSS, but 3-, 5-, 8- and 16-h treatment produced significantly increased expression and activity. NF- $\kappa \mathrm{B}$ DNA-binding activity was stronger at 30-min and 1-h LSS exposure than with control treatment. Pretreatment with $18 \mu \mathrm{M}$ SN50 efficiently inhibited NF- $\kappa B$ DNA binding activity. In the presence of SN50, MT1-MMP mRNA expression, protein level and activity were significantly attenuated (all $\mathrm{p}<0.01$ ). MT1-MMP mRNA expression, protein level and activity were inhibited by PD98059 (all $p<0.01$ ). To determine the relation between ERK1/2 and NF- $\mathrm{BB}$ with MT1MMP induced by LSS, HUVECs were incubated with PD98059 for $2 \mathrm{~h}$ before LSS. The level of phosphor-IKK $\alpha / \beta$ was reduced, whereas that of phosphor-I $\mathrm{B} \alpha \boldsymbol{\alpha}$ was increased as compared with no PD98059 treatment. As well, NF- $\mathrm{BB}$ DNA-binding activity was decreased. HUVECs were preincubated for $48 \mathrm{~h}$ with FAK siRNA and then treated with LSS. The shear stress-induced increase in MT1-MMP mRNA and protein level and activity was significantly inhibited (all $\mathrm{p}<0.01)$. As well, LSS-induced ERK1/2 activation was inhibited with 5 -min LSS exposure $(\mathrm{p}<0.01)$. To examine NF- $\kappa B$ DNA-binding activity, HUVECs were pretreated for $48 \mathrm{~h}$ with FAK siRNA before

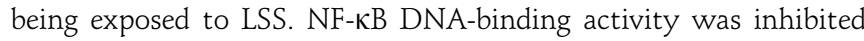
with FAK siRNA pretreatment. HUVECs were preincubated for $48 \mathrm{~h}$ with integrin $\beta 1$ siRNA and then underwent LSS. The shear stress-induced increase in MT1-MMP mRNA expression, protein level and activity was significantly inhibited (all $p<0.01$ ). As well, LSS-induced FAK and ERK1/2 activation was inhibited at 5-min

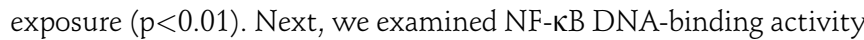
with 48-h integrin $\beta 1$ siRNA pretreatment before exposure to LSS; NF- $\kappa B$ DNA binding activity was inhibited by siRNA integrin $\beta 1$ pretreatment.

Conclusions MT1-MMP induced by LSS is involved in atherosclerotic plaque, via the integrin $\beta 1$-FAK-ERK1/2-NF-KB pathway.

\section{e0084 IMAGING OF VULNERABLE PLAQUE AND THROMBOSIS WITH MRI IN A RABBIT MODEL}

doi:10.1136/hrt.2010.208967.84

Zhao Quanming, Zeng Conghe, Feng Tingting, Zhao Xin, Ma Xiaohai, Zhang Zhaoqi. Beijing Anzhen Hospital

Objective Our aim is to investigate the feasibility of detecting vulnerable plaque and thrombosis by use of MRI.

Methods 24 male New Zealand White rabbits were divided into two groups: the atherosclerosis group (As group, $n=20$ ) and the normal control group ( $C$ group, $n=4$ ). After induction of atherosclerosis, MRI exams were conducted separately before and after the induction, triggering the plagues' disruption. The rabbits were then massacred to obtain data of pathology. The animals in the normal control group were fed a standard diet, and we performed MRI exam separately before and after, triggering the plagues' disruption too. After MRI exams, rabbits were massacred to obtain data of pathology. The in vivo imaging results of MRI were compared with the 